\title{
Técnicas Constructivas de la Arquitectura Moderna en México (1920 - 1950)
}

\author{
Constructive Techniques of Modern Architecture in Mexico (1920 - 1950) \\ Técnicas Construtivas de Arquitetura Moderna no México (1920 - 1950)
}

Wendy Margarita Montes Ponce

Arquitecta, PhD. en Arquitectura.

Universidad Autónoma Benito Juárez de Oxaca, México.

draarqwendymontes@gmail.com

(i) https://orcid.org/0000-0003-1962-4676

Otniel Josaft López Altamirano

Arquitecto, PhD. en Design.

Universidad Autónoma Benito Juárez de Oxaca, México.

otniel_digital@hotmail.com

(iD) https://orcid.org/0000-0001-7593-9715

Carlos A. Ortega del Valle

Lic. Artes Visuales, MSc. en Artes Visuales.

Universidad Autónoma Benito Juárez de Oxaca, México.

elkalor@hotmail.com

(iD) https://orcid.org/0000-0002-1047-7385

Recibido: agosto 20 de 2021

Aceptado: septiembre 24 de 2021

Publicado: septiembre 27 de 2021

\section{RESUMEN}

La Arquitectura en el México del Siglo XX estuvo provista de sistemas constructivos europeos y norteamericanos, heredados del quehacer decimonónico. Por tal motivo, el propósito del estudio expone el impacto de esos modelos edificatorios en los procedimientos de cimentación, reforzamiento estructural, cubiertas, servicios y cambio de escala. Así, pues, el método analítico condujo a relaciones específicas con base en las innovaciones constructivas, de composición estilística y patrones de funcionalidad. De manera que la aplicación de teorías y técnicas sobre Arquitectura, y su divulgación en revistas especializadas, formularon en un marco de treinta años el ambiente arquitectónico y urbano del modernismo mexicano.

Palabras clave: Sistemas Constructivos; Arquitectura Moderna; México; Teoría de la Arquitectura.

\section{ABSTRACT}

Architecture in the Mexico of the 20th century was provided with European and North American construction systems, inherited from the nineteenth-century work. For this reason, the purpose of the study exposes 
the impact of these building models in the procedures of foundations, structural reinforcement, roofs, services and change of scale. Thus, the analytical method led to specific relationships based on constructive innovations, stylistic composition, and patterns of functionality. Thus, the application of theories and techniques on architecture, and their dissemination in specialized magazines, formulated the architectural and urban environment of Mexican modernism within a thirty-year framework.

Keywords: Building Systems; Modern architecture; Mexico; Theory of Architecture.

\section{RESUMO}

A arquitetura mexicana do século $X X$ foi dotada de sistemas construtivos europeus e norte-americanos, herdados da obra do século XIX. Por este motivo, o objetivo do estudo expõe o impacto destes modelos de edifícios nos procedimentos de fundações, reforço estrutural, coberturas, serviços e mudança de escala. Assim, o método analítico conduziu a relações específicas baseadas em inovações construtivas, composição estilística e padrões de funcionalidade. Assim, a aplicação de teorias e técnicas sobre arquitetura, e sua divulgação em revistas especializadas, formou o ambiente arquitetônico e urbano do modernismo mexicano em um quadro de trinta anos.

Palavras-chave: Sistemas Construtivos; Arquitetura Moderna; México; Teoria da Arquitetura.

\section{INTRODUCCIÓN}

El desarrollo de la Arquitectura en México al iniciar el siglo XX consolidó la aplicación de los sistemas constructivos europeos y norteamericanos que se introdujeron durante el siglo precedente. Los profesionales de la construcción se dotaron de nuevos saberes que materializaron en obras de gran notoriedad arquitectónica y urbana. Los modelos tectónicos se adoptaron con fascinación, y condicionaron el diseño utilitario y estético modificando la apariencia de la ciudad de manera singular. Así, las transformaciones que experimentarían los procedimientos de cimentación, reforzamiento estructural, cubiertas y servicios, posibilitaron el cambio de escala.

Con base en lo anterior, el tema fue desarrollado a partir de seis apartados que en conjunto exponen el desarrollo que las técnicas constructivas de la Arquitectura Moderna en México, experimentaron durante 1920 a 1950. El propósito de la investigación se centró en relacionar los sistemas constructivos extranjeros y su introducción en el país, considerando que la mayoría de estos aún continúan vigentes en la arquitectura mexicana.

Los tres primeros temas exploran los sistemas constructivos empleados en algunos países europeos y en Norteamérica. En tal sentido, se demuestra el perfeccionamiento de estos en el proceso de industrialización. En tanto, los temas restantes exponen la aplicación de los sistemas constructivos del extranjero en la Arquitectura Mexicana posterior al periodo revolucionario. Por tal, se puntualizan las transformaciones que el diseño arquitectónico y urbano experimentaron, modificando sin precedente la vida cotidiana de la sociedad, motivo por el cual se gozaron de nuevos servicios que simplificaron la movilidad y el confort familiar.

\section{METODOLOGÍA}

La investigación se estructuró con base en el cuestionamiento sobre la vinculación entre los sistemas constructivos del extranjero y los aplicados en México en la primera mitad del siglo XX. Ello condujo a la selección del método analítico, pues posibilitó la evaluación de las relaciones implícitas entre los hechos, los actores y los productos arquitectónicos. El trabajo documental ha sido expuesto con apoyo de figuras que muestran detalles constructivos, edificios y contextos urbanos. Por tanto, el conjunto de información representa un ensamble fundamentado a través de diversos autores, más el criterio de quienes escribimos este ensayo. 


\section{RESULTADOS}

Métodos innovadores de industrialización. La integración de materiales y sistemas constructivos del siglo XIX permitió emprender novedosas propuestas constructivas, sólidas posturas teóricas, con base en procedimientos arquitectónicos que se fueron entremezclando con el contexto mexicano postrevolucionario. De manera que, con el diseño de nuevas propuestas tectónicas, plásticas y utilitarias, la vida cotidiana encontró satisfacción de viejos anhelos con base en la prosperidad y el confort.

Proyectos como la Rue Franklin, de Auguste Perret, de 1903, dieron evidencia de las noblezas -mecánicas y estilísticas- del hormigón estructural, demostrando la maleabilidad y ligereza que prometían los paneles de concreto (Strike, 2004, p. 108).

Desde luego, que el empleo del hormigón armado como elemento tectónico, igualmente había sembrado la confianza entre los constructores de principios del siglo XX. El Centro del Centenario de Breslavia (Jahrhunderthalle, Wroclaw) de Max Berg, edificado de 1911 a 1913 -ver Figura 1-, que fue construido bajo los principios de las estructuras cupulares de amarres nodales -salvando un claro de 67 metros de diámetro-, fue ejemplo de monumentalidad y tendencia como sistema constructivo (Strike, 2004, p. 108). Así, la regularidad con la que los arquitectos e ingenieros empezaron a desarrollar su quehacer edificatorio con base en este sistema cambió significativamente los conceptos constructivos, formales y utilitarios.

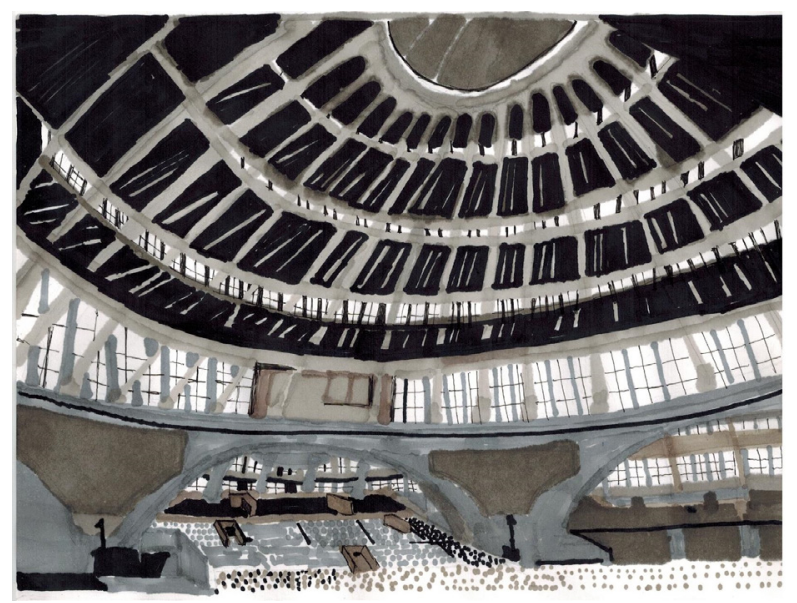

Figura 1. Jahrhunderthalle, Wroclaw (1911 - 1913). Fuente: Diana L. Martínez Cruz (2021).
Se trató de un tiempo en el que se teorizó sobre la economía arquitectónica. El perfeccionamiento y consolidación de nuevos sistemas constructivos hicieron de lo improbable un hecho arquitectónico probable. No obstante, el dominio de los procedimientos constructivos debió constituirse con ciertos titubeos antes de llegar al perfeccionamiento. El acceso a los manuales constructivos sirvió de apoyo en las tareas de los profesionales de la construcción, pues en ellos se describían los materiales, los recursos humanos, los procesos y las reglas de operación, aprendiendo de ellos los métodos innovadores de industrialización (Monjo Carrió, 2005).

La reproducción de prototipos arquitectónicos igualmente fue producto de la industrialización. Con el tiempo, cada proceso se identificó con base en un(a):

-Producción industrial de elementos constructivos, lo que se lleva a cabo en muchas ocasiones, sobre todo en los casos de "prefabricación".

- Proceso racionalizado y eficiente de ejecución en obra, con técnicas industriales que faciliten las operaciones y reduzcan la incidencia de la mano de obra, con una coordinación modular de los proyectos para facilitar el montaje con el uso de elementos de catálogo.

- Proceso industrializado de producción de unidades espaciales, que se montan en obra... (Monjo Carrió, 2005, p. 38).

La evolución de los sistemas constructivos, en las décadas posteriores a 1930, erradicarían procedimientos funcionales del siglo XIX. Se sustituirán las estructuras herméticas sostenidas a través de muros perimetrales posibilitando la apertura de generosos vanos, y se emplearon estructuras reticulares -vigas y columnas-. Se erradicaron los sistemas edificatorios pasivos, sujetos a trabajos de inercia, térmica, aireación, regulación de sombras, implementando recursos mecánicos, como los sistemas de acondicionamiento electromecánico. La incursión de materiales prefabricados, igualmente, abrió posibilidades en el quehacer arquitectónico. Los perfiles de hierro fueron protegidos contra la oxidación, en tanto que los materiales impermeabilizantes sobre las fachadas y cubiertas regularon la humedad producida por el intemperismo, a lo que se sumó la facturación de láminas de mayor resistencia e impermeabilidad, tanto para uso de cubierta plana como inclinada (Monjo Carrió, 2005). 
La garantía que los materiales debían ofrecer, en relación con su resistencia, durabilidad y funcionalidad, determinó agudas competencias entre los fabricantes, por lo que tuvieron que perfeccionar, a través de la experimentación, las propiedades alcanzadas, tratando así de evitar:

- Debido al fuego, pérdida de humedad con la consiguiente disminución de su capacidad resistente en los hormigones, deformación y pérdida de tenacidad en los perfiles metálicos y en las armaduras del hormigón armado.

- Debido a los agentes meteorológicos, oxidación y corrosión, en el caso de estructuras metálicas, carbonatación, lixiviación y meteorización del hormigón de recubrimiento, así como oxidación y corrosión de las armaduras, en el caso de elementos de hormigón armado (Monjo Carrió, 2005, p. 45).

Por lo anterior, John Brodie, en la ciudad de Liverpool, experimentó con paneles de hormigón prefabricado durante los primeros cuatro años del siglo XX. El sistema consistió en la colocación de módulos que conformarían los limites perimetrales de piso a techo. Cada una de las placas de hormigón que conformaban los módulos, estuvo reforzada con una malla de acero, cuyas uniones fueron resueltas con anclajes (Cornejo Vázquez, 2008, p. 15). Conocedor del sistema, Grosvenor Atterbury, en 1907, desarrollaría el perfeccionamiento de los elementos de hormigón aligerado, colocando en el mercado paneles aligerados; portantes que fueron empleados en varios de los edificios de la Unión Americana; materiales que ofrecieron mejores garantías y nuevos significados a los conceptos arquitectónicos: protección, higiene, iluminación, visibilidad, accesibilidad.

\section{Sistemas Constructivos Industrializados.} Dentro de los aportes más significativos en la Arquitectura, con base en sistemas constructivos industrializados, se identifican los sistemas dependientes producidos en cadena de montaje en sitio. En consecuencia, a ello el ferrocarril, el vehículo de carga y los barcos formularon redes de abastecimiento de materiales, maquinaria, equipo y otras materias primas y recursos humanos especializados.

Esos aportes estimularon proyectos experimentales por todo el mundo. El conjunto de viviendas que el Departamento Británico de Investigación Científica e Industrial de la Junta de Agricultura y Pesca, se construyó, entre 1919 y 1920, en Amesbury (Wiltshire), como uno de esos productos. Para ello, se emplearon bloques de Cob (similar al adobe), tapial y bloques de hormigón adicionado con margas, reproduciendo casas de dos niveles:

... La vivienda de hormigón tenía dos pisos, muros de hormigón armado realizados en obra, un primer forjado a base de viguetas prefabricadas en perfil en $\mathrm{T}$ y unas cercas de cubierta prefabricadas que sostenían un cerramiento estanco tipo Pudlo de 50 milímetros... Pese a que la mayoría de estos sistemas piloto no lograron ningún avance, unos cuantos sí llegaron a alcanzar una producción limitada... contribuyeron a largo plazo a la evolución de las técnicas de prefabricación y de los sistemas constructivos (James., 2004, p. 130).

La empresa Waller perfeccionó la facturación de placas de hormigón armado. El diseño del muro para exteriores se reforzó al aplicar dos placas externas prefabricadas con hormigón armado de tres milímetros. La hoja interior de hormigón de clínquer era de cincuenta milímetros; separando ambas mamparas por una cámara de 75 milímetros. La montura de estas placas se desarrollaba in situ, a través de soportes facturados en obra, empleando aleros y vigas de hormigón -ver Figura 2-. Y aun cuando el sistema no llegó a ser rentable, alrededor de 500 casas fueron fincadas bajo este sistema en Pool y en Leeds.
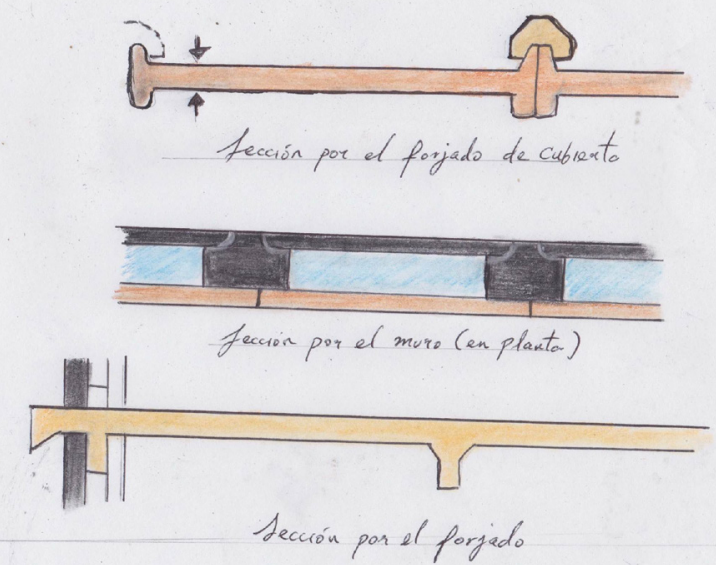

Figura 2. Sistema Waller, 1920.

Fuente: Carlos A. Ortega del Valle (2021). 
En paralelo, el sistema Dorlornco, de la firma Dorman \& Long, industrializó la producción de estructuras de acero. Los elementos ferrosos revestidos con mortero de 38 milímetros sobre un enlistonado metálico nervado y bloques de clínquer de 50 milímetros de espesor y acabado de yeso, garantizaban la resistencia y compatibilidad entre sus componentes. Bajo esta alternativa constructiva se edificaron 3000 viviendas tipo, identificadas como casas de acero Atholl (James, 2004, p. 132) -ver Figura 3-. No obstante, la desafortunada pesadez y tosquedad que poseía este sistema constructivo evitó su desarrollo comercial.

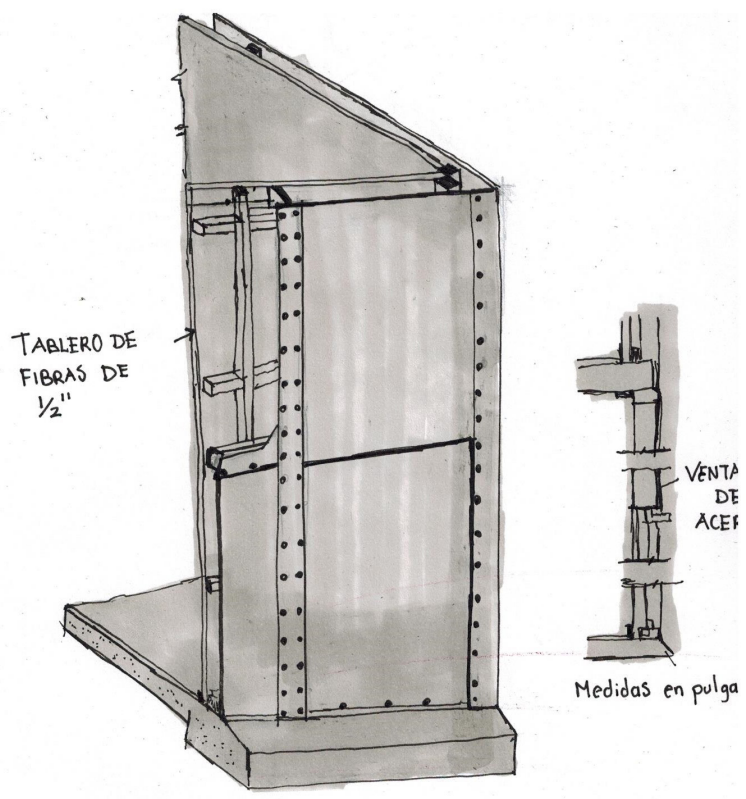

Figura 3. Detalle de las viviendas tipo Atholl -1920-. Fuente: Diana L. Martínez Cruz (2021).

Los progresos que los fabricantes en sistemas prefabricados habían alcanzado experimentaron los efectos de la crisis posbélica en la segunda década del siglo XX. La producción de casas prefabricadas gradualmente entró en su propia recesión. Empero, continuaron diseñando novedosas soluciones habitacionales. Las escasas publicaciones administradas por arquitectos, como lo fue la Unit One, cuestionaron la función social del arquitecto, el sentido de la Arquitectura y las raíces de la disciplina. Los innumerables fundamentos, discursos y manifiestos sobre el tema destacaron la importancia de los sistemas constructivos compuestos con base en materiales prefabricados. Se destacó el empleo del hormigón armado como principal componente para la factura de estructuras sin puntales, en serie:

Le Corbusier suponía que los entramados estructurales Domino serían fabricados en serie por un contratista y luego la casa sería terminada por otro contratista distinto, usando para ello ventanas, puertas $y$ tabiques normalizados, y consiguiendo así las diferentes distribuciones en planta requeridas para cada cliente (Strike, 2004, p. 135).

Desde luego que los aportes del Arquitecto Charles Édouard Jeanneret merecen mayor exposición, por lo que volveremos a su quehacer arquitectónico más adelante. En paralelo, el prototipo de la "casa de acero", de Georg Muche y Richard Paulick, daba gala de su estabilidad al difundirse su sistema constructivo. Se trató de la edificación sustentada por cimentación de hormigón, estructura de acero y cerramiento con base a planchas exteriores de acero Siemens de 3 milímetros de espesor, aislante, cámara de aire, torfoleum pro tablero, placas de mortero y cartón de yeso. Sistema edificatorio que será mejorado por Walter Gropius en la factura de dos casas, financiadas por el ayuntamiento de Stuttgart, a razón de la exposición de viviendas Weissenhofsiedlung de 1927 (Strike, 2004, p. 140).

La tipología de cimentación igualmente tuvo su desarrollo que fue de gran apoyo para el quehacer constructivo de los arquitectos e ingenieros en los primeros años del siglo pasado. La madurez alcanzada en el sistema de pilotes y zapatas de cimentación, se debió al sinnúmero de correcciones que exigió la factura de puentes edificados en el periodo decimonónico. La consulta de tratados sobre soluciones tectónicas, actualizaba a los profesionales sobre los materiales y las técnicas que debían emplear para la edificación de un correcto soporte estructural. Cierto es que el perfeccionamiento de la cimentación siempre fue un asunto de primer orden para los constructores, empero no se erradicaron los sistemas del emparrillado y pilotes de madera; dando continuidad a las tres variantes tradicionales:

- La constituida por un emparrillado apoyado sobre pilotes hincados, al igual que en las ya mencionadas cimentaciones de "Beaugency". - La formada por un emparrillado, colocado directamente sobre el terreno en el arranque del cimiento y por un pilotaje perimetral que, a modo de tablestacado, bordeaba el 
mencionado cimiento, le servía de recintado y, cuando existía agua, protegía su fábrica frente a la socavación.

- La construida con un emparrillado, colocado directamente sobre el terreno en el arranque del cimiento, y con pilotes hincados en los espacios definidos por la cuadricula de vigas que constituían dicho emparrillado (Gamallo, 1998, p. 254).

Las primeras pruebas de penetración fueron ampliamente sugeridas en el siglo XVII por Bullet; quien argumentó que cada pilote alcanzaría su optimización dependiendo del tipo de suelo en el que estuviera activo. Sostuvo también que la longitud de cada pilote era dependiente del elemento del que fuera sustentante. Gautier, al sumarse al fundamento de Bullet, acentuaba la impronta de la capacidad portante entre los grupos de pilotes. Así, se constituyó el sistema constructivo de pilotes de cimentación. No obstante, el desgaste que las puntas de los pilotes experimentaban por causas del empuje y la fricción, fue una de las soluciones que con mayor atención atendieron los estudiosos del sistema de cimentación.

Los tratados recomendaban, en consecuencia, el reforzamiento del ápice, endureciendo las puntas y las cabezas por calentamiento. Mientras que especialistas como Belidor sugerían tallar la punta en forma de diamante, dando una longitud de una y media, a dos veces el diámetro del pilote (Gamallo, 1998). Otro de los elementos que requirió de una solución permanente fue el perfeccionamiento de la maquinaría. El Martinete de Hinca que diseñara Turriano en el siglo XVI, se fue modernizando con la integración de cuerdas que fijaban la posición de la maza --ver Figura 4-. La energía continua que requerían estos mecanismos, igualmente fueron evolucionando hasta sustituir la fuerza mecánica de la bestia por la mecanización industrializada:

Cualquiera que fuera la clase de energía utilizada, no cabe duda de que uno de los mayores problemas que se planteaban en la hinca de pilotes era el de la liberación de la maza. Parece que esta cuestión no se resolvió hasta mediados del siglo XVIII, cuando se inventaron los primeros mecanismos de castañuelas, también llamados "de tijera", que liberaban la maza de manera automática, mediante el movimiento guiado de unas ruedas sobre unas guías, que funcionaban a modo de levas... (Gamallo, 1998, p. 283).

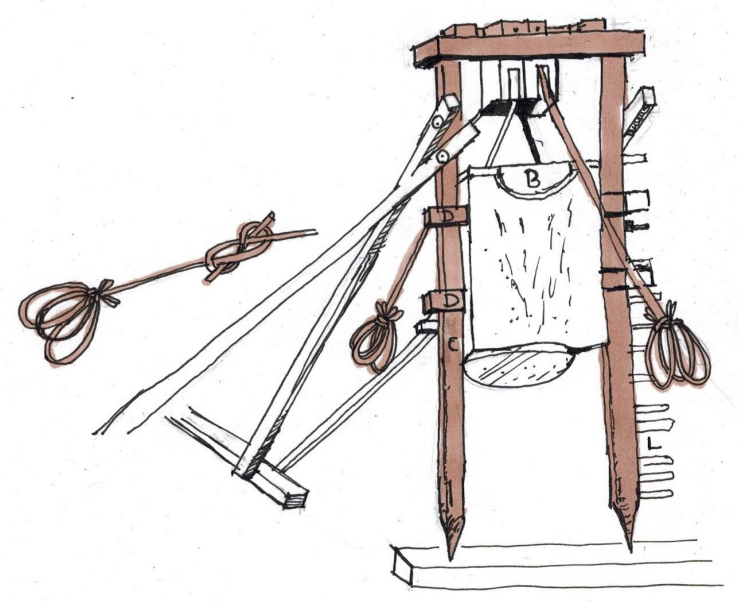

Figura 4. Martinete de Hinca, diseño de Turriano (S. XVI). Fuente: Diana L. Martínez Cruz (2021).

Sería bajo este sistema sobre el cual en la maquinaria construida por Boulton y Watt se resolvería el problema de la liberación manual del pestillo y golpeteo; así, el instrumento industrializado producido representó la primera maquinaria de vapor de ocho caballos de fuerza, funcional para pilotes de cimentación, puesta en marcha por John Rennie en 1804 -ver Figura 5-.

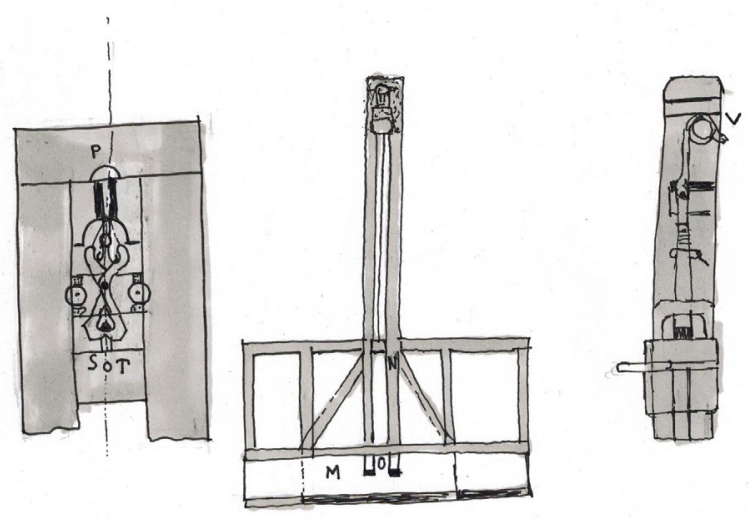

Figura 5. Mecanismo de Castañuela para la liberación de la maza (S. XIX).

Fuente: Diana L. Martínez Cruz (2021).

La estabilidad vertical de los pilotes no resultaba una tarea absolutamente sistemática; las características de los terrenos exigió de los ingenieros expertos el desarrollo de pruebas de mecánica de suelos, 
publicándose, en consecuencia, las primeras tablas de dureza y resistencia de suelo. De esta manera, la cimentación por pilotes adquirió su propia clasificación: pilotes y emparrillados, pilotes y cajón de fondo, pilotajes y hormigón, pilotajes en terrenos fangosos, palizadas metálicas, palizadas de hormigón armado, cimientos sobre pilares aislados. La participación literaria de los ingenieros en materia de divulgación de sistemas constructivos para cimentación resultaría muy activa sobre la segunda década del siglo XX. Caso concreto serán las producciones intelectuales del ingeniero José Eugenio Ribera, académico corresponsal de la Academia de Ciencias de España.

La seguridad técnica que los estudios de mecánica de suelos, para el diseño del sistema de cimentación correspondiente a cada proyecto, representó para los arquitectos e ingenieros nuevas posibilidades edilicias. La Arquitectura entró en una etapa de desarrollo de escalas mayores. La ruptura de la altura uniforme de las construcciones de la ciudad no tardó en manifestarse. De este modo, los recursos constructivos se integraron a las necesidades circunstanciales del periodo de entreguerras. Fue un pre requisito el diseño y construcción de una arquitectura práctica, funcional y resistente. Los profesionales en construcción de la primera mitad siglo XX demostraron, a través de sus obras, las noblezas que la arquitectura había alcanzado.

Edificios Verticales Modernos y Salubres. El crecimiento vertical de los inmuebles se distinguió por el diseño de la apariencia del paralelepípedo. La estilización plástica se centró en el efecto óptico que el inmueble causaría en el espectador. La liviandad, la colosal verticalidad, la gama de texturas y acabados, significaría uno de los retos más complejos a resolver por los profesionales, toda vez que el sistema de soporte - cimentación-, era ya solo un evento de selección.

La edificación del sistema de marco rígido con elementos estructurales de acero, experimentó un desarrollo importante durante las últimas décadas del decimonónico. La arquitectura de H. P. Berlage, Alfred Messel y Otto Wagner, en Holanda, Alemania y Austria, respectivamente, figuraron como hitos en el quehacer constructivo del mundo (Behne, 1994, p. 25). Los edificios modernos y sanos, tal como se les calificó, fueron producto de una escrupulosa evaluación funcional, generando, en consecuencia, nuevos conceptos a dominar. Exponiendo Otto Wagner en 1895, en Die Baukunst unserer Zeit: -
Comprensión escrupulosamente exacta y satisfacción completa de la finalidad hasta el último detalle.

Elección acertada de los materiales de ejecución (fácilmente disponibles y manejables, y económicos en relación con su durabilidad.

Construcción económica y fácil.

La forma que resulta de estas premisas (se compone sola y es siempre fácilmente comprensible) (Behne, 1994, p. 30).

En paralelo, Messel promovió el diseño por escaparate en los primeros cinco niveles, asignando a los pisos restantes elementos relacionados con la publicidad de la época, solución que colocaba a los elementos estructurales metálicos en segundo plano, de manera que se enfatizó la plástica con base a la multiplicidad de los ejes en sentido vertical, provocando un efecto visual que, en perspectiva, fundía sus elementos en una sola pieza.

La demanda constructiva que los edificios de hierro y hormigón experimentaron en el ocaso del siglo XIX, desarrolló el crecimiento de la empresa The Phoenix Company Handbook y la Carnegie Pocket Companion. Los rascacielos edificados representaron símbolos de competitividad y riqueza. Exponentes del sistema de cajas o retícula estructural -esqueleto de acero-, fueron William Le Baron Jenney, autor de edificios mayores a doce plantas. Por su parte, Henry Hobson Richardson, Burnham y Root, desarrollaron inmuebles mayores a veintidós plantas. En tanto que Louis Sullivan edificó obras mayores a diecinueve pisos, llegando a materializar con este sistema el edificio L. Empero, C. Smith, en 1914, edificaría una construcción vertical de cuarenta y dos plantas. El sistema de esqueleto estructural sería, por tanto, empleado por sus noblezas que implicaban:

Estructuras metálicas (esqueletos o armazón de hierro) que, entre otras cosas, permitirá realizar edificios con gran altura.

Uso del pilar de hormigón como soporte o cimiento. Será la solución al desafío de construir sobre un suelo arenoso y fangoso.

Ventanas extendidas horizontalmente por toda la fachada (con las dimensiones que se desee, dado que ya no serán necesarios los llamados muros de carga):

Posible eliminación de los muros de carga (gracias a esta estructura metálica) Desarrollo del ascensor eléctrico 
Con respecto al exterior, se suprimen los elementos decorativos (tan habituales en la arquitectura artística de finales del siglo XIX). Se apuesta por superficies lisas y acristadas. Predominan las líneas horizontales y verticales.

Atractivas fachadas de mampostería. (Vaquero, 2010, p. 15).

La sustitución de las placas de mampostería por el cristal, como recurso estético de la fachada puso al alcance de los arquitectos e ingenieros el sistema de estructura metálica autoportante. El muro cortina es el resultado de una búsqueda constructiva decimonónica. En colaboración con Adolf Meyer, Walter Gropius, consolidaría el sistema de Curtain Walls, al diseñar anclajes y forjados más prácticos -ver Figura 6-, capaces de transmitir el peso propio entre cerramientos:

Todas las paredes que no son sustentantes, y que han sido utilizadas en las tradiciones más distintas de la construcción, se pueden designar, en un determinado sentido como Curtain Walls... poseen tanto las propiedades de una pared (inmóvil, pesada, definitiva, como las de una cortina (movible, ligera, provisional)...

Sin embargo, las paredes-cortina, en un sentido moderno, presentan una categoría especial de muros exteriores no sustentantes. Constan de elementos modulares que se van repitiendo, fabricados en serie y que se montan en el mismo lugar de la obra y a los que corresponden las funciones de la separación de lo interior y lo exterior, de la defensa contra las influencias exteriores, de aislamiento térmico y acústico y de regulación de las vistas exteriores, luz y aire... (Diccionario llustrado de la Arquitectura Contemporánea, 1970, p. 84).

El simbolismo que se le atribuyó al sistema representó ser su mejor recurso publicitario. Identificado como una apariencia ideal para inmuebles de oficina, por sus espacios diáfanos ver Figura 7-. El doble techo que en ellos se manejó se aprovechó para invisibilizar los ramales o líneas de las instalaciones que requerían los servicios modernos.

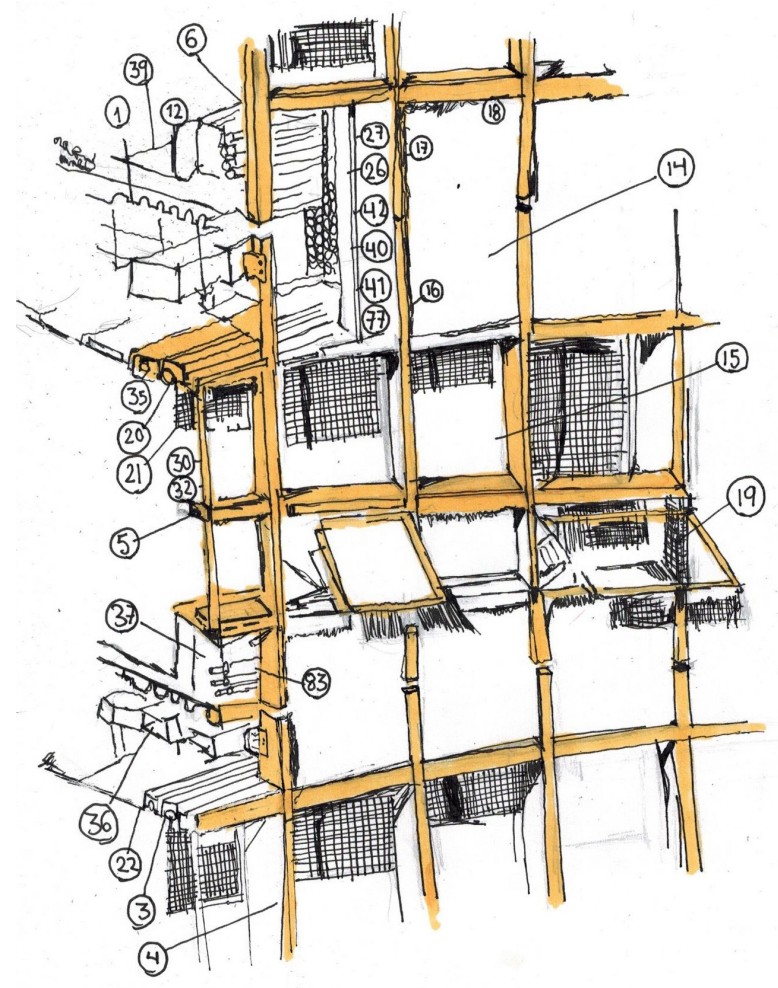

Figura 6. Elementos Generales del Muro Cortina clásico. Fuente: Diana L. Martínez Cruz (2021).

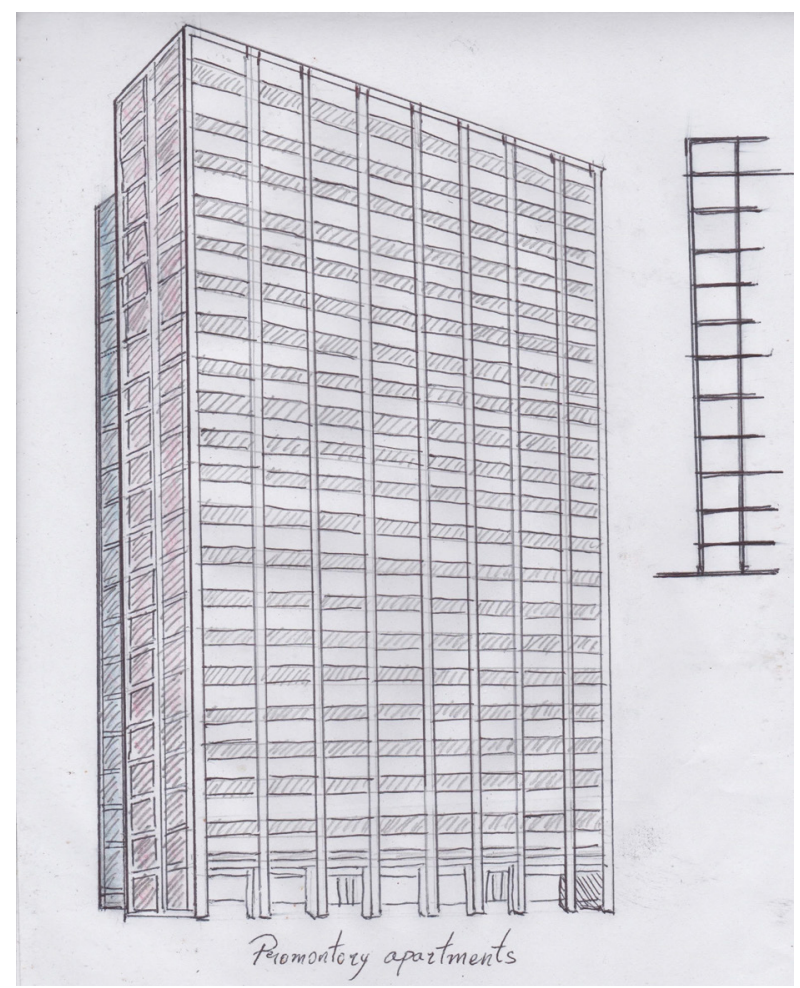

Figura 7. Promontory Apartments

Fuente: Carlos A. Ortega del Valle (2021). 
Las propiedades tectónicas estéticas y funcionales del sistema fueron aprovechadas por los constructores para reproducir obras homogéneas. Notable es el trabajo de Louis Sullivan al respecto. El aspecto exterior de la construcción estaba en relación con el uso espacial interno de sus rascacielos. Comercios, oficinas y viviendas fueron los espacios predominantes de sus obras. De modo que se fincaron edificios de alto consumo energético, al emplear para el confort instalaciones controlables, como el aire acondicionado. En tanto que el uso del muro cortina inhabilitó la abertura de las ventanas y promovió el uso de sistemas de inyección aire para aminorar los efectos de la climatización (Cueva, 2011).

El éxito inmobiliario de los rascacielos, solicitó la participación de arquitectos e ingenieros, la vinculación de ambos saberes - el arte y la ciencia-, centrados los mismos propósitos, desarrolló nuevas habilidades de planeación, composición y procedimientos edificatorios. El urbanismo vertical modificaría sustancialmente el entender de convivencia, pluralidad e individualidad del sujeto en la primera mitad del siglo XX. Estados Unidos llevaba la avanzada con su arquitectura del "Estilo Chicago". La arquitectura europea, desarrollaría lo propio "...Europa en movimientos como el racionalismo de la Bauhaus, el funcionalismo de Le Corbusier, el neoplasticismo de Mies Van der Rohe y el constructivismo ruso" (López Jiménez, 2013, p. 19). Pareciese entonces que la Arquitectura se definiría a partir de dos bloques ideológicos. Las búsquedas de los arquitectos europeos estuvieron centradas en las emergentes demandas arquitectónicas que se generaron durante los tiempos de posguerra. La vivienda, la salud, la educación, los espacios para la diplomacia, el comercio y los negocios, representaron una motivación para nuevas propuestas en el quehacer arquitectónico.

El talento con el que los arquitectos europeos y americanos resolvieron los problemas constructivos se proyectó en diversos postulados y manifiestos que delinearon las respuestas tecnológicas, utilitarias y plásticas de la Arquitectura. La teoría definirá la praxis, y la praxis definirá la teoría, como un proceso holístico que serviría de orientador para la búsqueda particular de los profesionales en formación o de las naciones de diferentes latitudes. De esta manera, la producción en serie, la diversidad de sistemas constructivos y multiplicidad de los materiales abrió oportunidades para una arquitectura de lenguaje conceptual:
Fue tal la fascinación por la producción en serie y la revolución constructiva que los nuevos materiales ofrecían que, primero en la Bauhaus y después en los CIAM, se gestó la propuesta de una Arquitectura que respondiera a las necesidades básicas de la población y se eliminará la ornamentación y todo aquello que no cumpliera una función. Para Le Corbusier uno de los objetivos de la arquitectura era ordenar a la población a funcionar de forma sincronizada como lo hacía una máquina, como lo expresó en la frase "La vivienda no es más que una máquina de vivir" (López Jiménez, 2013, p. 17).

De acuerdo con los datos consultados, el primer rascacielos europeo data de 1920; se trataba del edificio Telefónica. Este se desplantaría $89.3 \mathrm{~m}$, contando con catorce pisos, poseedor de gran novedad visual en la ciudad de Madrid. De esta forma será España uno de los países que desarrollará la explotación de alto rendimiento del suelo. No obstante, sobre la tercera década del siglo XX, la construcción de edificios de gran altura era una labor recurrente en diversas naciones. Tal como fue el caso de la Unión Soviética al aprobarse por Stalin el proyecto de la edificación de ocho rascacielos (edificados sólo siete), con los que se conmemoraría en 1947, el VIII centenario de la fundación de Moscú.

En realidad los arquitectos en Estados Unidos sintieron poca afinidad con las formulaciones teóricas de los arquitectos Walter Gropius, Mies Van der Rohe o Le Corbusier. La confianza que la arquitectura vertical del periodo de la Escuela de Chicago había producido en ellos, sumado a la empatía de las recetas inmobiliarias que sostenían el capitalismo norteamericano, posibilitó el perfeccionamiento de los sistemas constructivos para la factura de rascacielos:

...la evolución de la arquitectura norteamericana entre 1900 y 1925 siguió, con pocas excepciones, sus propias vías. En lugar de relacionarse con los movimientos modernos europeos, recurrió al repertorio de modelos históricos y revistió los edificios altos, tan innovadores desde el punto de vista de la ingeniería técnica, con una fachada historicista... Aun cuando en Chicago Sullivan inicio la arquitectura moderna de los rascacielos, otros arquitectos norteamericanos mezclaban sin ningún rubor elementos modernistas con un lenguaje formal afín al clasicismo, al románico y al gótico (Tietz, 1999, p. 42). 
El desarrollo de los rascacielos estandarizó muchos de los procesos edificatorios y aumentó la complejidad técnica y tecnológica. Razón por la cual se crearon corporativos dedicados exclusivamente a la facturación de edificios verticales. Los diseñadores y profesionales se fueron agrupando en equipos de especialistas de diferentes saberes para la exitosa construcción, con lo cual se garantizó la planeación y ejecución proyectos. El arribo de Mies Van der Rohe a Estados Unidos, renovó el lenguaje plástico, tecnológico y utilitario de la arquitectura. La absoluta transparencia de sus inmuebles a cuatro fachadas vinculó las actividades internas con la dinámica exterior de la ciudad. Cada uno de sus prismas representa la innovación del sistema constructivo del muro cortina y la recuperación del ladrillo como elemento soportante, que se fusiona con las propiedades tectónicas de los perfiles estructurales -ver Figura 8-:

...el modelo de Mies para el perímetro de bandas de ladrillo y cristal del Reichsbank derivaba de la tradición industrial alemana de Fachwerkbauten, donde el armazón de acero visto se combinaba con un relleno de ladrillo y cristal. Este sistema constructivo derivaba a su vez de la tradición industrial alemana de Fachwerkbauten tradicional de estructura de madera, y en la segunda mitad del siglo XIX el armazón de acero vernáculo y modificado se convirtió en el modelo común para cobertizos baratos... presencia vernácula, el Reichsbank de Mies presenta un muro cortina continuado más que un armazón y un sistema de relleno... será decisivo en la formación de su obra americana (Frampton, Bozal , \& Calatrava, 1999, p. 183,184).

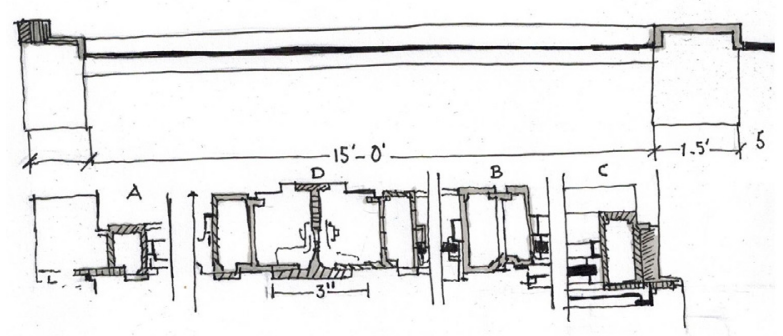

Figura 8. Promontory Apartaments, detalle del relleno de alumino.

Fuente: Diana L. Martínez Cruz (2021).

El uso de secciones metálicas como elementos soportantes verticales, que desarrolló Van der
Rohe, dejó como manifiesto la resistencia de cada uno de estos diseños con base en las propiedades de sus ángulos. Así, los elementos verticales que tradicionalmente poseían forma circular o cruciforme fueron intercambiados soportes en forma de " $\mathrm{H}$ " o "I". Estructuralmente, la columna debía actuar como un elemento de sostén; mientras que desde el aspecto plástico debía aportar a la belleza del volumen. La apariencia resultante se identificó como estilo Internacional. Y merece mencionarse, el soporte vertical de dos secciones en canal invertido, unido a través de soldadura para formular la caja vacía; recubriendo la sección con enladrillado aparente.

Arquitectura Mexicana al iniciar el Siglo XX. Los sistemas constructivos que en la Arquitectura Mexicana se estaban ejecutando en los primeros años del siglo XX resultaron ser similares a los que se habían desarrollado en Europa y Estados Unidos. Los primeros edificios construidos con base en los sistemas tectónicos de esqueletos de fierro y cimentación de concreto fueron inmuebles de uso comercial. La producción nacional de acero facilitó el suministro del material a la capital del país, haciendo innecesaria la importación de este. En consecuencia, la demanda de consumo en el cemento -material que ya se producía en el país unas décadas antes de la primera fundidora de Monterrey-, estimuló la fundación de otras plantas cementeras. Lo que implicó la integración de otros materiales para el uso de técnicas estructurales, o para el uso de detalles decorativos; tales como el ladrillo, la cerámica, el vidrio, aluminio, cobre (Cardenas Valdéz, 1999).

Las edificaciones que se ejecutaron a partir de la segunda década en México adaptaron sistemas constructivos y procedimientos técnicos del extranjero, principalmente norteamericano y europeo. La limitada comercialización de los perfiles estructurales conllevó al diseño de estructuras mexicanas. Los materiales se fueron diseñando con base en el dominio de dos saberes, el del profesional de la construcción, y el del maestro herrero. De esta manera, la producción de los elementos estructurales en hierro se desarrolló a partir de cálculos de diseño estructural. El hierro fundido o forjado, en doble $\mathrm{T}$, angular, en $\mathrm{U}$, redondo o cuadrado, laminado, acanalado o torsión, se consolido como el material primario en las edificaciones de todo el país: 
Durante los años veinte, se generaliza el uso de estructuras de hierro en construcciones industriales, gracias a la creación de la Compañía de Aceros Monterrey en 1917. La primera estructura completa que se realizó fue para la fábrica de hilados y tejidos La Victoria (1922), al año siguiente para la Escuela Industrial de Orizaba, el mercado de Villahermosa, las fábricas de San Rafael, La Hormiga y la Cervecería Modelo, donde el espacio arquitectónico adquirió nuevas dimensiones físicas y estéticas (Cardenas Valdéz, 1999, p. 28).

El material fundamental para la construcción de la cimentación fue el acero. El poco uso del sistema de pilotes se relaciona por los altos costos que ello implicaba. Sería entonces, el recurso del sistema de emparrillado de fierro el que dio solidez a los inmuebles mayores a dos niveles. Es de hacerse notar, que los sistemas estructurales estaban sujetos a estudios sobre mecánica de suelo, siendo el notable ingeniero mexicano Hermion Larios, uno de los primeros profesionales especialistas en la materia. La cimentación entonces era calculada en relación al tipo de suelo, materiales que le resultaban compatibles al trabajo mecánico, tomando en consideración la teoría de M.L. Engers M.A.S.C.E., a la resistencia dinámica por fricción basada en la fórmula del Engineering News, el número de trabes y losas de máxima rigidez, constituían la plataforma hidrostática que evitaba hundimientos. La cimentación por pilotes, resultaría ser un sistema idóneo para edificios de mayor altura. Este sistema trabaja a partir de postes, que deprecian su resistencia a partir del fenómeno de flotamiento trabajo a fricción-. Estos elementos estructurales se diseñaron con base a diámetros de 25 a $35 \mathrm{~cm}$. Elaborados con madera de árboles de eucalipto, encinchados de hierro en los extremos, como medio de reforzamiento (Cardenas Valdéz, 1999).

Las cubiertas laminares de concreto ya se empleaban con regularidad en 1910. Las losas de concreto con vigas voladas, arcos y losas cilíndricas, se perfeccionaron gracias al trabajo del ingeniero Modesto C. Rolland:

... [Rolland] construye en 1925 el estadio de Jalapa, Veracruz, con alerones de $11.00 \mathrm{~m}$. En 1927 Federico Mariscal construye el edificio para la Durkin con arcos de concreto armado de $14.00 \mathrm{~m}$. de claro. Américo Schawrs, ese mismo año, construye la fábrica de focos eléctricos Águila Nacional, con una bóveda de concreto armado de $20.50 \mathrm{~m}$. de claro y un espesor variable de 10 a 15 centímetros (Cardenas Valdéz, 1999, p. 32).

El método del cálculo de Hennebique, determinaba el diseño estructural de otros elementos tectónicos. El espesor de las losas se determinaba en relación al claro y a las cargas que debían soportar, considerando ante todo que el concreto no resistía trabajos a tención y que los esfuerzos eran equivalentes en las zonas comprimidas. Los momentos estáticos eran calculados considerando las particularidades internas y externas del elemento. Esté método permitió que el cálculo de losas se diseñara por secciones al igual que su armado. El diseño de columnas con base al sistema Hennebique, se estructuró a partir de un cuarteto de barras de acero de diámetro variable, y estribos de reforzamiento sobre los extremos de la sección.

\section{Sistemas y Procedimientos Constructivos Posrevolución Mexicana. Al término de la} Revolución Mexicana (1910-1917), el conjunto de sistemas y procedimientos constituido por pilotes, zapatas, losas de cimentación, columnas y losas de concreto para entrepisos y azoteas formuló el sistema de marcos rígidos de concreto. Este recurso técnico sustituyó los marcos rígidos de acero. El concurso que convocó, la Cámara Nacional de Cemento en 1929, con motivo del primer centenario de la invención de este material en Inglaterra, evidenció la capacidad de dominio que los arquitectos habían desarrollado, al edificar obras de exquisita calidad. Evidencia de ello fue el resultado de la premiación:

Los premiados fueron: el Ingeniero José A Cuevas y los arquitectos Bernardo Calderón Caso y Manuel Ortiz Monasterio, por el proyecto del edificio de La Nacional, Compañía de Seguros sobre la vida, S.A., obra urbano de primera categoría en que el concreto se dejó expuesto en función abiertamente decorativa. Este edificio fue el primero de esta categoría que se hizo en México, por lo cual despertó gran Interés y constituyó un paso firme de la ciencia de la cimentación aplicada en la ciudad (Cardenas Valdéz, 1999, p. 80).

El edifico de La Nacional, representó así la introducción de nuevas posibilidades constructivas. El estudio de suelo determinó la conveniencia del sistema de cimentación por pilotes sobre un terreno pantanoso, perfeccionando el sistema de flotación que se había aplicado en obras menores. Disponiendo para ello pilotes de madera de 35 a 25 
centímetros de diámetro. La predilección de postes de madera se debió a la propiedad de ligereza, y su capacidad de conservación debajo del agua. En este sentido, el edificio de 1929, encargo consignado a la firma Monasterio y Calderón, impulsó los aportes tecnológicos del concreto reforzado.

El edificio Ermita, construido por el Arquitecto Juan Segura en 1930, representa otro de los ejercicios tectónicos de innovación -ver Figura 9-. La cimentación fue resuelta con base a vigas continuas que se ligan por pilotes. Los muros que se desplantan sobre esta estructura poseen un rodapié de piedra basáltica, reforzada con solera de concreto armado de $10 \mathrm{~cm}$., de espesor. Tanto los muros divisorios, como los de carga se facturaron con tabique rojo recocido; amarrando la estructura con columnas, trabes, vigas y cerramientos estructurales de concreto armado y losa de concreto. La integración de materiales ornamentales como el mármol, granito, bronce y latón, requirieron un sistema de montajes, que perfeccionó los ensambles de los maestros herreros.

La Nacional, y el Ermita, edificios altos de esta época son el resultado de conquistas que la arquitectura tuvo al emplear materiales nuevos, en ambas la presencia extensiva del cemento lo mismo en la estructura portante que en acabados exteriores, se consideró un argumento clave para su propio desarrollo constructivo (Cardenas Valdéz, 1999, p. 100).

La torre Latinoamericana, edificada en 1948; implicó la participación de diversos profesionales. La cimentación fue diseñada para compensar las vibraciones del subsuelo; solución tectónica que se le debe al ingeniero consultor el Doctor Leonardo Zeevaert. Para lo cual, el profesional dispuso la distribución de 361 pilotes de concertó ButtomBottom, de esta manera:

La cimentación está constituida por una retícula de trabes de concreto que soportan las columnas y trasmiten la carga a las losas haciendo coincidir la resultante del peso total del edificio con el centro de gravedad del conjunto de los pilotes. El volumen total de concreto empleado en la cimentación fue de 2,750 m". Hay trabes de cimentación de 1.20 $\mathrm{m}$ de ancho por $4.50 \mathrm{~m}$ de peralte, reforzadas hasta con 110 varillas de $1 \frac{1}{2} 2$." Se usó un concreto impermeable para contener con efectividad el empuje del agua de abajo hacia arriba, que representa el 54\% del peso total del edificio" (Cardenas Valdéz, 1999, p. 104).

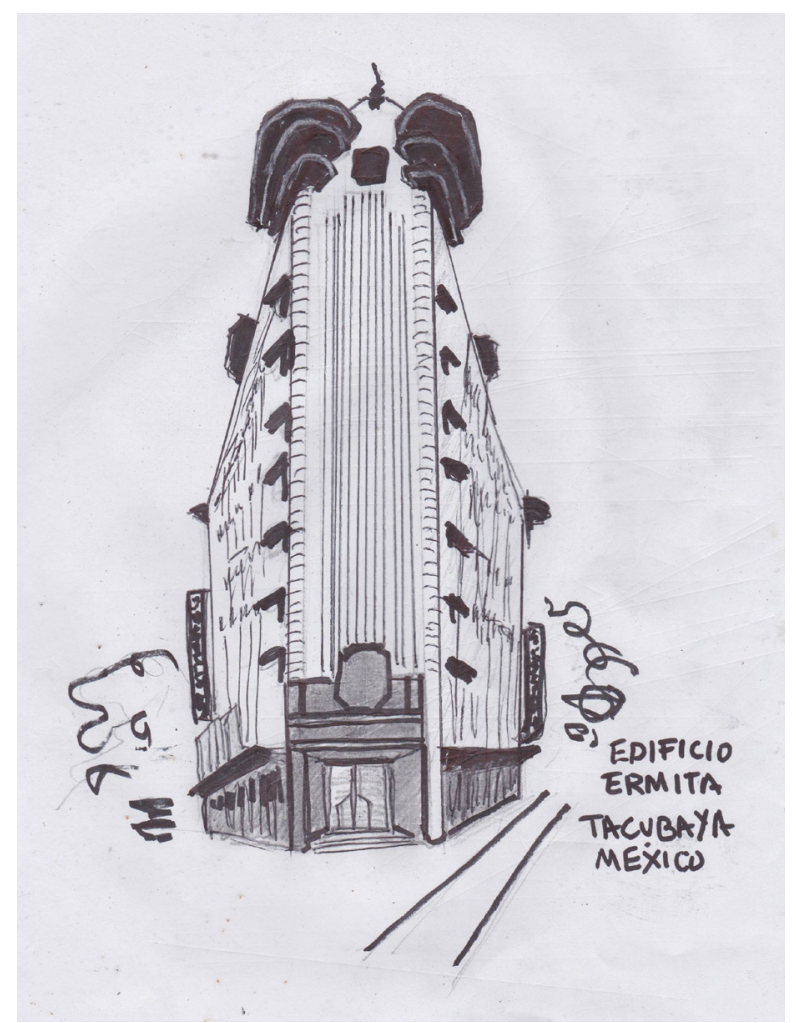

Figura 9. Edificio Ermita, 1930

Fuente: Carlos A. Ortega del Valle (2021).

En el edificio se emplearon los principios del sistema de esqueleto de acero. Respondiendo de manera ortodoxa a la tectónica del estilo Internacional. La selección de los materiales se determinó a partir de sus noblezas, como lo fueron la ligereza, la economía, y la factibilidad montante.

Aportaciones Técnicas aplicadas a las Estructuras en México

El empleo de los sistemas constructivos-descritos líneas arriba- que hicieron los arquitectos en México permitió el desarrollo de un sinnúmero de obras arquitectónicas que enriquecieron no solo los aportes tectónicos de la cuarta década del siglo XX -ver Figura 10-. La síntesis de esas contribuciones tecnológicas está representada en el proyecto de Ciudad Universitaria -Universidad Nacional Autónoma de México (UNAM)-. Proyecto que fue resultado de un concurso coordinado y ejecutado bajo la coordinación general de los arquitectos Mario Pani y Enrique del Moral, y la organización ejecutiva del Arq. Carlos Lazo. Mención aparte requiere el arquitecto Félix Candela, sus aportaciones técnicas revelan su 
interés por el estudio de matemáticas aplicadas a las estructuras. Su exploración particular se materializó en el desarrollo de las membranas de concreto armado, sujetas a las geometrías diversas, innovadoras y elegantes. Muchos fueron los ejercicios constructivos que al respecto hiciera Candela. Cada uno de ellos producto de una estructura modular que posibilitó la edificación de amplios claros. Se trata de cascarones de concreto con espesor entre 4 a $7 \mathrm{~cm}$., soportados por escasos apoyos verticales -ver Figura 11-. Bajo una estética armoniosa, de apariencia ligera, y óptimas corrientes de aire e iluminación. Generando con ello adecuados microclimas naturales.

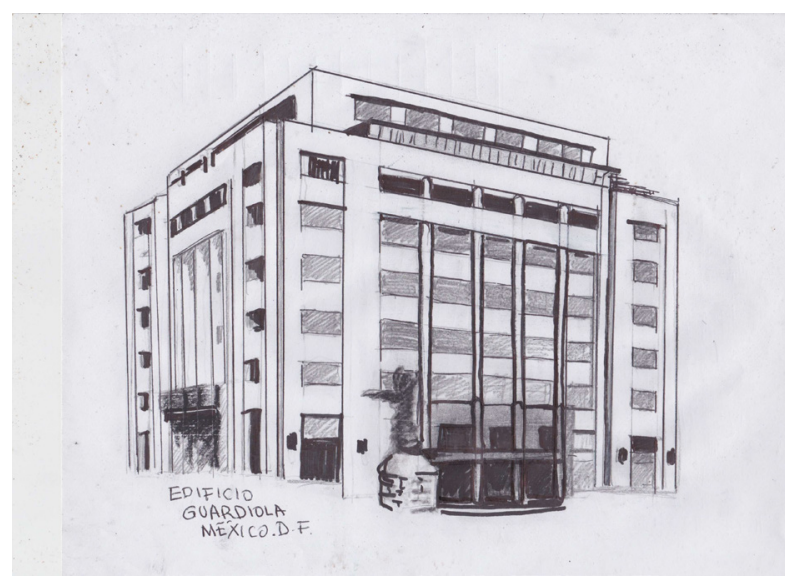

Figura 10. Edificio Guardiola, 1947.

Fuente: Carlos A. Ortega del Valle (2021).

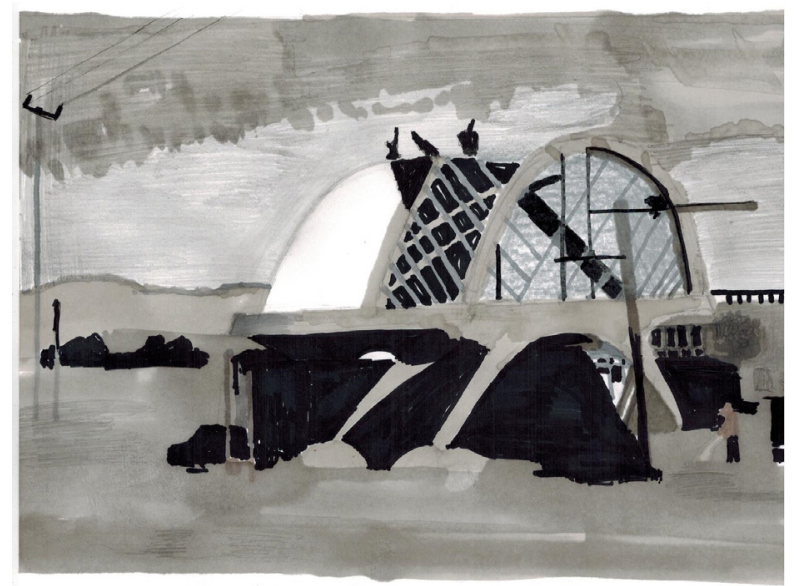

Figura 11. Pabellón de Rayos Cósmicos(UNAM), 1951. Fuente: Diana L. Martínez Cruz (2021).

El impulso inicial que tuvo este sistema constructivo materializó obras como el pabellón de rayos cósmicos (UNAM), estaciones del metro en México, el Palacio de los deportes o la fábrica embotelladora para la marca Bacardí. El bajo costo de mano de obra y el bajo consumo de materiales como el acero, implicaban una inversión financiera del $50 \%$ en comparación con otras soluciones estructurales. Empero, factor importante que determino el desuso de este recurso tectónico fue, el alto costo de la madera que se empleaba para el diseño de la cimbra (Carmona Aparicio, 2008, p. 85).

\section{Conclusiones}

Los arquitectos en México de la primera mitad del Siglo XX se entusiasmaron con los aportes constructivos que se integraban al quehacer edificatorio. La sistematización de los procesos les resultó conveniente, pues emplearon con amplia destreza los modelos tectónicos industrializados.

La composición arquitectónica se constituyó a partir de patrones constructivos, relaciones espaciales y formulaciones estilísticas influenciadas por las obras de los arquitectos europeos más difundidos en las publicaciones circulantes en el país.

Se trata de una arquitectura que empleó nuevos paradigmas teóricos y prácticos. En consecuencia, se produjo un cambio de escala predominantemente vertical. Arquitectos como: Carlos Obregón Santacilia, Juan Serrano, Mario Pani, Enrique del Moral, Augusto H. Álvarez, Enrique Yañez; representan esa generación de profesionales, constructores de la arquitectura erguida.

La aplicación de sistemas mecánicos contribuyó al cambio de escala. Los edificios verticales se dotaron de servicios dependientes de la fuerza motriz, otorgando a estas valoraciones superiores; como fuera el caso de ser calificados como elegantes, funcionales y modernos.

El sistema de cimentación con base a pilotes y zapatas de cimentación sustituyó a los sistemas tradicionales de cimientos de mampostería; pues otorgó confianza en los arquitectos. Algunas obras en las que se aplicaron pilotes de cimentación serían: la torre Latinoamericana y el edificio Ermita en la Ciudad de México.

La integración a la arquitectura del sistema por cadena de montaje redujo el tiempo de ejecución de obra, pues se trataba de piezas prefabricadas ensambladas 
en sitio. Ejemplo de ello fueron las placas de hormigón empleadas en cubiertas y entre pisos.

El hormigón armado, ampliamente utilizado en las obras verticales requirió de instrumentos normativos. Los resultados de las pruebas de mecánica de suelo se difundieron a través de tablas de dureza y resistencia del suelo; formando parte de los requisitos constructivos.

El uso del sistema de esqueleto estructural brindo oportunidades de ligereza y extensión de altura, abertura de los vanos y eliminación de muros divisorios interiores. La planta libre que de ello se generó permitió la modulación espacial y flexibilidad funcional.

El urbanismo vertical, modificaría sustancialmente el entender de convivencia, pluralidad e individualidad del sujeto en la primera mitad del siglo XX. Por ello, la vivienda, la salud, la educación, los espacios para la diplomacia, el comercio y los negocios, representaron una motivación para nuevas propuestas en el quehacer arquitectónico.
La arquitectura vertical en México, inspirada en la Escuela de Chicago impulsó el mercado inmobiliario. En consecuencia, los profesionales implicados consiguieron el perfeccionamiento de los sistemas constructivos para la factura de rascacielos.

Al reproducir los géneros arquitectónicos del extranjero, los arquitectos en el país consumieron materiales fabricados en México. La demanda de materiales de acero, cemento, ladrillo, cerámica, el vidrio, aluminio y cobre, implicó la innovación de técnicas estructurales y detalles decorativos.

La arquitectura de la primera mitad del Siglo XX está sintetizada en el conjunto de edificios de la Universidad Nacional Autónoma de México. Las aportaciones técnicas revelan el estudio de las matemáticas aplicadas a las estructuras, la función espacial en congruencia con la utilidad, y la identidad nacional a través de la composición plástica.

\section{REFERENCIAS}

Behne, A. (1994). 1923 La construcción funcional moderna. España: Serbal.

Cardenas Valdéz, M. I. (1999). Efecto de la evolución tecnológica en la arquitectura mexicana en la primera mitad del Siglo XX. México: Universidad Nacional Autónoma de México.

Carmona Aparicio, C. (2008). Estudio sobre el diseño del Paraboloide Hiperbólico. México.: Universidad Nacional Autónoma de México.

Cornejo Vázquez, T. I. (2008). Diseño Estructural de una casa modular de dos niveles con elementos prefabricados de acero y placas de concreto. México: Universidad Don Vasco A. C.

Cueva, E. (04 de abril de 2011). Obtenido de Instituto de Ciencias de la Construcción Eduardo Torroja: http:// upcommons.upc.edu/pfc/bitstream/2099.1/4129/1/Memoria.pdf

Diccionario Ilustrado de la Arquitectura Contemporánea. (1970). España: Gustavo Gili.

Frampton, K., Bozal , A., \& Calatrava, J. (1999). Estudios sobre cultura tectónica. Poéticas de la construcción en la arquitectura de los siglo XIX y XX. España: Akal.

Gamallo, G. (1998). La Evolución de las Cimentaciones en la historia de la arquitectura, desde la prehistoria hasta la primera revolución industrial. España: Universidad Politécnica de Madrid.

James., S. (2004). De la construcción a los proyectos. La influencia de las nuevas técnicas en el diseño arquitectónico, 1700 - 2000. Barcelona: Reverte. 
López Jiménez, A. M. (2013). Urbanismo vertical, el rascacielos en la ciudad de México. México: Universidad Nacional Autónoma de México.

Monjo Carrió, J. .. (2005). La Evolución de los sistemas constructivos en la edificación. Procedimientos para su industrialización. Consejo Superior de Investigaciones Científicas. Vol. 57, 499-500.

Strike, J. (2004). De la construcción a los proyectos. La influencia de las nuevas técnicas en el diseño arquitectónico 1700 - 2000. Barcelona: Reverte.

Tietz, J. (1999). Historia de la arquitectura del siglo XX. España: Könemann.

Vaquero, A. (2010). La Escuela de Chicago y La Vanguardia Americana. Apuntes para la clase de Historia de la Arquitectura IV. Honduras: Universidad de San Pedro de Sula. 